\title{
IoT Based Smart Home Automation System
}

\author{
Aakriti Tyagi, BE, Savitribai Phule Pune University in Pune India, aakriti.tyagi5@gmail.com
}

Smita Deshmukh, BE, Savitribai Phule Pune University in Pune India, smitadeshmukh1996@gmail.com

Gayatri Dindokar, BE, Savitribai Phule Pune University in Pune India, gayatridindokar1@gmail.com

Shraddha Kale, BE, Savitribai Phule Pune University in Pune India, shradhhakale00@gmail.com

Mayur Karale, BE, Savitribai Phule Pune University in Pune India, mayurkarale34@gmail.com

Bhagyashree Dhakulkar, Assistant Professor, Savitribai Phule Pune University in Pune India,

bhagyashree.dhakulkar@gmail.com

\begin{abstract}
As technology advances, it makes life easy and straightforward by solving many of the real-life problems which are faced by people in their daily life. Various types of technologies are used to improve the life of humans. Hence, this paper has used one of the technologies to ease the life of humans i.e. IoT. This paper represents IoT (Internet of Things) for the smart home automation system. There are many proposed systems which make the home easy to live in. But this paper tells about the newer advanced system. This paper tells the usage of motion detection technique to perform light on/off, MQ2 sensor for gas detection, machine learning for facial expression detection, motor for facade opening/closing, motor for room rotation and last the website "ThingsBoard" to collect the whole data at one place. The main goal of this paper is to provide full security with innovation at home.
\end{abstract}

Keywords - Android, Face Detection, Face Recognition, MCP3208 IC, Motor, Raspberry Pi, Relays, Sensor, ThingsBoard.

\section{INTRODUCTION}

Our Future is in our hands so let's make it better and proper. In this world of technology, everyone has a very busy life and one can think of living in a comfort zone rather than getting it stressed. So why cannot everyone use technology to make things better? Home Automation is the first approach to this, which has become and will become a trend in 21st and the upcoming century. Home Automation is a term used to describe the working of all the household amenities and appliances together and control it from our daily use or things especially smartphones, tablets, or a computer having Internet connectivity. According to the statistics report, it has been recorded that from 2005 to 2015 the increase in Internet users is been increased from 1,024 to 3,207 million and will increase more in the future. Mobiles, smartphones, and laptops are the means from which one can use the Internet at any time and from anywhere. So, if it opens a door that connects us to various home appliances in our daily use then anyone can control his/her own home.

Internet of Things is a concept where each device is assigned to an IP address and through that IP address, anyone makes that device identifiable on the internet. The Internet is an evolving entity. It started as the "Internet of Computers." Research studies have forecast explosive growth in the number of devices that will be connected to the Internet. IoT is having the potential to change the lifestyle of peoples. In our daily life, people prefer automatic systems rather than any manual systems. The major elements of the IoT based smart home automation system are Raspberry pi, Relay, Sensor, and the Motor. Smart Home automation is often defined as a mechanism removing human interaction in various domestic processes and replacing them with programmed electronic systems. Ultimately it is a system that aims to heighten the quality of life with the automation of household appliances that may be controlled over the Internet or Telephone.

The Internet can be used in home automation to make many decisions like saving energy, protection, and safety. Even if a person is at a great distance the user can manage and monitor all the home appliances.

\section{LITERATURE REVIEW}

Automation is increasing day by day like a competition between players on a field. To cope up with this race people have to advance their thinking. Rather than writing their thoughts on paper, people should have to implement it. The author presents a method to develop the smart lowcost home automation system which is designed using IoT. With the help of this system, all the home appliances and electronic machines can be controlled and observed through a website very easily. The metering method of a home can also be supervised using this system which is explained in [2] and with the slight change in [2] the author control and monitor the system using an embedded micro-web server, which is also used with IP connectivity for accessing and controlling devices and appliances 
remotely using Android-based Smartphone app which is explained in [13].[3] explains the home automation system which can be effectively used to control and monitor home appliances using the internet. Due to its various advantages, home automation is gaining more and more popularity day by day because of its ability to ensure security and make life much easier. In the system, Arduino will be interfaced with multiple sensors that can measure temperature \& humidity, light, motion, and so on. The data collected by the various sensors are stored and pattern analysis is done on the stored data which tells the user at which time the appliances are usually on or off so that they can be automatically controlled without any human intervention by observing the regular usage pattern. The user can also turn on/off any appliance remotely using the internet.

Similarly, like in [3] the author used an open-source system which is the Arduino platform. It is also a typical cyber-physical system and consists of input, output, and energy monitoring functions explained in [12].

Day by day increase of technology, everyone then introduced to a new method of face recognition. In [1] the author explains that human emotions are natural expressions that people tend to make naturally, instead of any conscious effort that is accompanied by the reflexing of facial muscles. Some of the common emotions are Happy, sad, surprised, anger and stable (normal) which a human face can make according to the different situations one may find itself in. The objective of the project is to be an affordable and efficient product. Artificial Intelligence \& Digital image processing technology used to make the system in python. As the system also recognizes the identity card, this is a simple feature wherein the camera installed is trained in such a way that it firstly focuses on the card and recognizes its shape and color.

In [4] explains the program of face recognition application using the fisherface method by utilizing GUI applications and databases are used in the form of a Papuan facial image. Image recognition is done using the fisherface method which is predicated on the reduction of face space dimension using the Principal Component Analysis (PCA) method, and apply Fisher's Linear Discriminant (FDL) method or also referred to as Linear Discriminant Analysis (LDA) method to get the feature of image characteristic.

With some improvement, everyone is then introduced to ZIGBEE. [8] The author proposed a system in which a web or an android application is used by the users to give instructions to the systems/ appliances from remote locations. This system can make use of a number of communication methods like Wi-Fi, GSM, Bluetooth, and ZigBee. Different controlling devices and configurations are often found in existing systems. Such systems have been found already in many places for a wide variety of applications and [10] also demonstrates the control of home appliances wirelessly using a computer and Zigbee wireless communication protocol. The author proposed a system through which one can control the home appliance like a refrigerator, fan, and air conditioner, etc. by sending a command through the computer. Inputs are going to be given from the PC through serial communication. By this technique, can also control all electrical appliances within a room in a building. [9] also proposes an efficient implementation for IoT (Internet of Things) used for monitoring and controlling the home appliances via the World Wide Web. They can communicate with home automation networks through an Internet gateway, utilizing low power communication protocols like Zigbee, Wi-Fi, etc. This project aims at controlling home appliances via smartphones using $\mathrm{Wi}-\mathrm{Fi}$ as a communication protocol and raspberry pi as a server system. The user will move directly with the system through a web-based interface using the web or internet, whereas home appliances like lights, fan, and door lock are remotely controlled through the easy website. By this, the paper provides a climbable and price-effective Home Automation system.

Many houses use raspberry pi which is controlled by android application. [6], [16] introduces a smart home concept that improves the standard of living at home. The paper is intended to control electrical appliances in a home or office using an android application. The main control system implements wireless technology to provide remote access from a raspberry pi or Arduino BT. The paper mainly focuses on the monitoring and control of smart home remotely and providing security, when the user is away from home. The paper is intended to control electrical appliances and devices in the house with relatively low-cost design, user-friendly interface, and ease of installation. This paper differentiates itself from others as it has its software level application to control the home appliances. In this paper, an android phone is used to control the various parameters. Android phone proves to be the main advantage compared to a personal computer, as it is portable. As technology increases like voice command. In [7],[14] provides home security and emergency system to be activated. The main motive of home automation and security system is to help handicapped and old aged people which can enable them to manage home appliances and alert them in critical situations. This project put forwards the implementation of home automation and security system with the help of Arduino microprocessor and Android phone. Home appliances are connected to the microprocessor and communication takes place between the Arduino and Android mobile device or tablet via Bluetooth. It presents the design and implementation of an automation system that can monitor and control home appliances via an android phone or tablet. 
Making technical development and socio-economic implications of the Raspberry Pi as a Learning Tool in a developed country. [11] explained the potential for providing a demo tool capable of teaching science, engineering, and computing in the developing world. This study explains the planning, developing, and manufacturing of a prototype solution for educational based usage within the schools in Uganda whilst considering the social implications of implementing such solutions. During the planning and manufacture of the prototype, software and hardware were developed also as testing performed to define the performance and limitation of the technology. This study showed that it is possible to develop viable modular-based computer systems for educational and teaching purposes. In addition to science, engineering, and computing; this study considers the socioeconomic implications of introducing EPI within developing countries. From a sociological perspective, it's shown that the success of EPI depends on understanding the social context; therefore, a next phase implementation strategy is proposed.

By considering the health of humans working in an office, there is also a new technique which is explained in [5]. The author said that facades are the most strategic and visible part of the building which leads to an improvement in appearance and environmental performances in buildings. Facades play a big role in the quality of a building. It forms the barrier between the interior space and therefore the outside climate. This means that the facade is the medium through which the interaction takes place between the activities, inside and outside. The paper will discuss the concept and therefore the importance of dynamic facades consistent with their design and kinds, implementations, current challenges, and climate impacts.

In [15] explains that the system is based on the LabVIEW software and can act as a security guard of the home. The system can monitor the temperature, humidity, lighting, fire \& burglar alarm, and have an infrared sensor which guarantees the family security. The system also has an internet connection to monitor and control the house equipment's from anywhere in the world. This paper presents the hardware implementation of a multiplatform system for house automation using LabVIEW.

\section{PROPOSED SYSTEM}

The proposed system follows the following features:

1) Raspberry $\mathrm{Pi}$

2) Devices controlled through raspberry pi.

3) When any person enters into the room the light automatically turns on and vice versa.

4) Detect the Gas at home.

5) The song plays automatically according to the person's facial expression using the camera.
6) The room rotates up to 360 degrees and gives the view of all the directions.

7) The dynamic facade protects the glass window/building from sunlight.

8) ThingsBoard collects and stores all the data from the appliances and can be access and control from anywhere in the world.

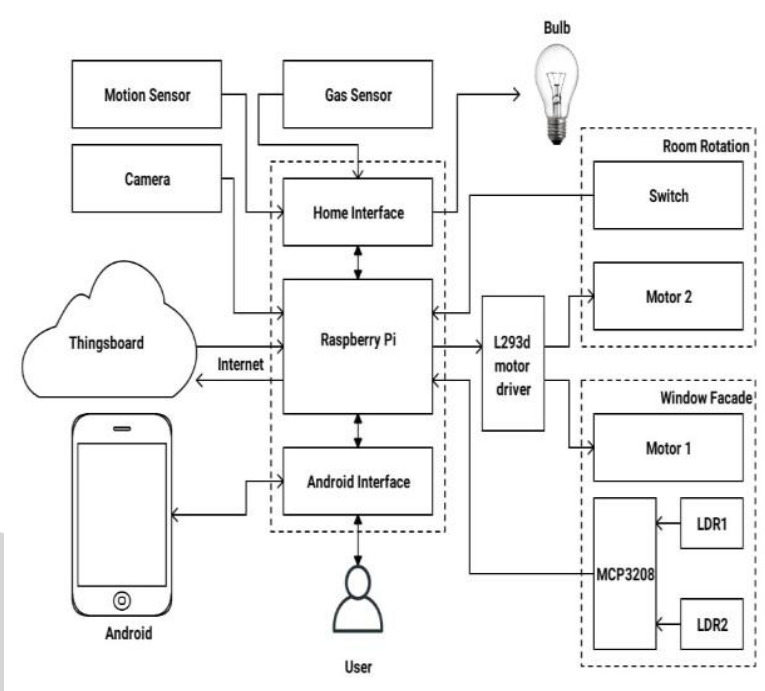

Fig:1 System Architecture

Motion and gas sensors are connected to the raspberry pi. They give their data to the home interface then the data flows from home interface to the RPI after it analyzes the data it then again sends the data to the home interface and it blows the bulb.

In the same manner the camera clicks the photo of the expression of the person and sends the data to the RPI and then it starts playing the song on basis of expression of the person.

We can also perform light on/off manually through the Android interface and also gas leakage message is sent to the user's mobile.

Now in room rotation we press the switch and the signal goes to the RPI which sends analyzed data to the motor to rotate the room.

And in the facade, the LDR sensor sends the detected sun rays to the MCP 3208 IC. After converting the analog signal to the digital signal, it sends the signal to the RPI. Which then sends the signal to the motor which makes the facade open/close.

And at last the Thingsboard is connected to the RPI. This board collects all the home appliances data at one place so that anyone from anywhere can access this data.

\subsection{Raspberry pi:}

\subsubsection{GB SD card}

3.1.2. A USB Micro power supply 
3.1.3. Ethernet connectivity

3.1.4. Pins for an interface: 40 pins

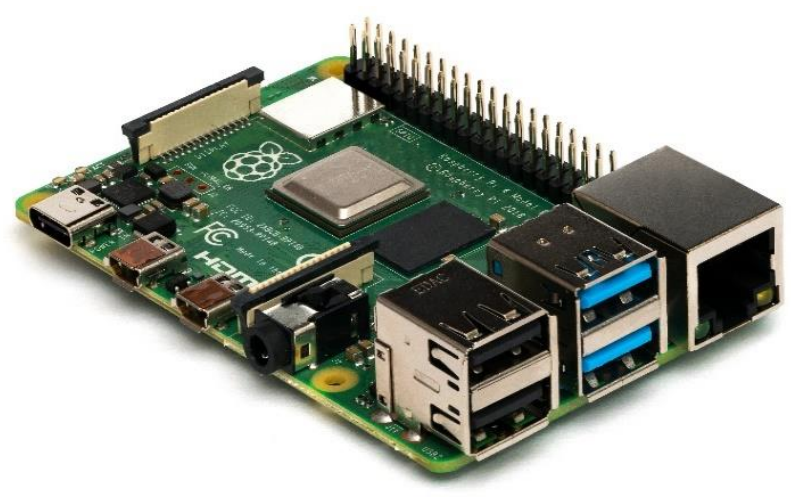

Fig: 2

The Raspberry $\mathrm{Pi}$ is a small credit card size computer developed in the United Kingdom for controlling electronic devices. The original $\mathrm{Pi}$ has a single-core $700 \mathrm{MHz}$ CPU with just 256MB RAM, and the latest model has a quad-core $1.4 \mathrm{GHz}$ CPU with 1GB RAM. All over the universe, people use Raspberry $\mathrm{Pi}$ to learn programming skills, build hardware projects, used in building a smart home and even use them in industrial applications.

The Raspberry Pi is a very cheap computer that runs on Linux, but it also provides a set of GPIO pins that allows the user to control electronic devices for physical computing and to explore the Internet of Things (IoT).

\subsection{Relay:}
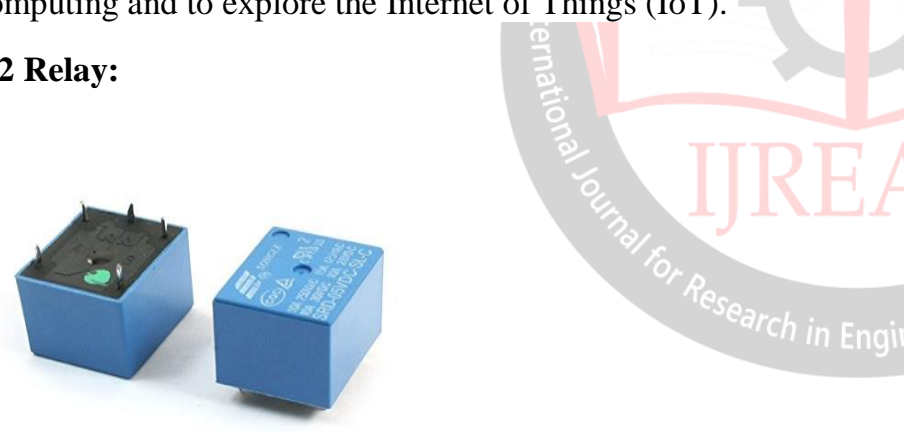

Fig: 3

A relay is an electrical switch that uses an electromagnetic system to move the switch from the on to off position instead of manual action. It takes a relatively small amount of power to switch on or switch off using a relay. The AC unit probably runs off on 220VAC at around 30A. That's 6600 Watts! A relay switch can be divided into two different parts such as input and output. The input section has a coil that generates a magnetic field when a small voltage coming from an electronic circuit is applied to it. This voltage is called an operating voltage. Commonly used relays are available in a different configuration of operating voltages like $6 \mathrm{~V}, 12 \mathrm{~V}$, etc. The output section consists of contactors which connect or disconnect mechanically for the switch on and switch off. In a basic relay, there are three contactors: normally open, normally closed, and common. At no input state, the COM is connected to normally close. When the operating voltage has applied the relay, the coil gets energized and the COM changes contact to normally open.

\subsection{Gas sensor:}

\subsubsection{BLOCK: Input}

\subsubsection{TYPE: MQ-2}

\subsubsection{ANALOG/DIGITAL: Analog or digital}

\subsubsection{PINS FOR INTERFACE: 6 Pins}

\subsubsection{PHOTO/CIRCUIT SYMBOL:}

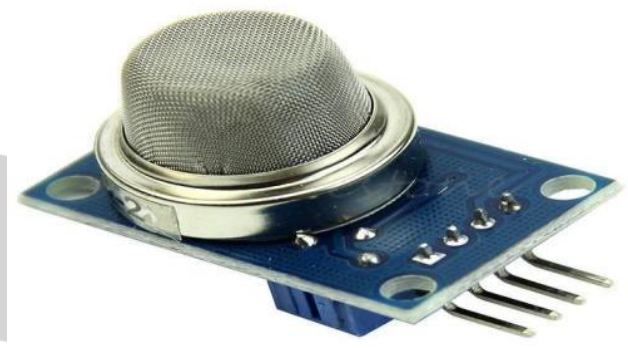

Fig: 4

MQ2 gas sensor is an electronic sensor used for sensing the number of gas molecules in the air such as LPG, propane, methane, hydrogen, alcohol, smoke, etc. MQ2 sensor is also known as chemiresistor. It contains a sensing material whose resistance changes with the time when it comes in contact with the gas molecules. This change which occurs in the value of resistance is used for the detection of the gas. MQ2 sensor is a metal oxide semiconductor type gas sensor. Concentrations of gas in the air is measured using a voltage divider network present in the gas sensor. This sensor works on a $5 \mathrm{~V}$ DC voltage power supply. It can detect gas molecules in the concentration of range between 200 to $10000 \mathrm{ppm}$.

This sensor contains a sensing element for sensing the gas, mainly aluminum-oxide, coated with Tin dioxide, enclosed within a stainless-steel mesh. The sensing element has six connecting pins attached to it. Two pins are responsible for heating the sensing element, the other four are used for output signals. Oxygen gets adsorbed on the surface of sensing material when it is heated in air at high temperature. Then donor electrons present in the tin oxide are attracted to these oxygen molecules, thus preventing the current flow. Now current can flow through the sensor, which generates analog voltage values. These voltage values are used to measure the concentration of gas.

\subsection{Infrared (IR) Sensor:}

3.4.1. BLOCK: Input 
3.4.2. TYPE: IR Sensor

3.4.3. ANALOG/DIGITAL: Analog or digital

\subsubsection{PHOTO/CIRCUIT SYMBOL:}

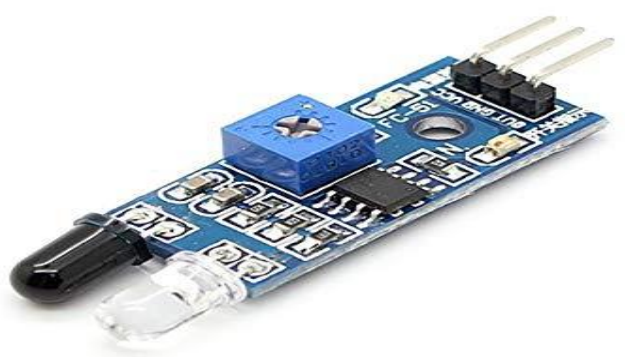

Fig: 5

An IR sensor is an electronic device that detects IR radiation falling on it. Proximity sensors, contrast sensors, and obstruction counters/sensors are some applications involving IR sensors. An IR sensor consists of two parts such that the emitter circuit and the receiver circuit. This is collectively known as the photo-coupler. The emitter is the IR LED and the detectors are the IR photodiode. The IR photo-diode is sensitive to the IR light emitted by the IR LED. This is the working principle of the IR sensor. The type of incidence can be direct incidence or indirect incidence. In direct incidence, the IR LED is placed in front of the photodiode with no obstacle in between them. In indirect incidence, both the diodes are placed side by side with an opaque object in front of the IR sensor. The light from the IR LED hits the opaque surface and reflects a return to the photodiode.

\subsection{Stepper motor:}

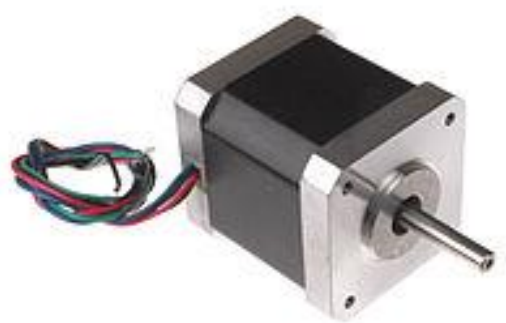

Fig: 6

A stepper motor is also known as a step motor, it's a brushless DC motor. It can divide a full rotation of objects into many equal parts. The stepper motor is an electromagnetic device that converts the digital pulses into mechanical or analog shaft rotation. The advantages of stepper motors are low cost, high reliability, high torque at low speeds, and they are simple, rugged construction that can operate in almost any environment. The drawback of the stepper motor is the resonance effect often exhibited at low speeds and decreasing torque with an increase in speed.

\subsection{Motor driver L293D:}

L293D is a popular 16-Pin Motor Driver IC. As the name suggests it is mainly used to drive motors. A single L293D IC is capable of running two DC motors at the same time and also the direction of these two motors can be controlled independently.

\subsection{Light turns on/off automatically:}

Here, the system uses an Infrared (IR) sensor for automatic on/off of the light. IR sensor receives a ray (radiation) from an object or particular person and according to the sensed ray/energy the IR sensor works. And the light on/off process occurs. When a person enters the room, the IR sensor senses the radiation and sends detected value to the Raspberry $\mathrm{Pi}$, and light will turn on and vice versa action happens while leaving the room.

\subsection{Gas Detection:}

As everyone knows MQ2 gas sensor detects different gases like LPG, propane, methane, hydrogen, alcohol, smoke, etc. Here, the system used it for LPG leakage detection. MQ2 gas sensor is connected to the raspberry pi. Have to set some threshold values so as if gas leaks above that threshold value sensor will detect it. If the gas intensity is high than the threshold value, the notification is sent to the user's phone that there is a leakage of gas at home.

\subsection{Song will play according to the person's facial expression:}

Here, the concept of face detection and extracting the facial expressions/emotions of the person in front of the camera is used. The camera will connect to the Raspberry pi to capture the input (human face) and then it stores the image of the person and process the image to the raspberry pi. It then changes from RGB (3D) to grayscale (2D). And then the song will play according to the expression of the person. Grayscale (2D) blurs the background (things/objects) and takes only the face.

This paper used the Fisherface algorithm for face detection, feature extraction, and face recognition.

\section{FisherFace:}

Fisher face is one of the popular algorithms used in face recognition and is widely believed to be superior to other techniques, such as eigenface because of the effort to maximize the separation between classes within the training process. The purpose of this research is to determine the concept of face recognition application using the fisherface method by utilizing GUI applications and databases that are utilized in the form of a CK facial image. The image recognition technology uses the fisherface method to predicated on the reduction of face space dimension using the Principal Component Analysis 
(PCA) method, then apply Fisher's Linear Discriminant (FDL) method or also referred to as Linear Discriminant Analysis (LDA) method to get the feature of image characteristic. The algorithm utilized in the method for image recognition is fisherfaces algorithm while for identification or matching face image using minimum Euclidean.

Generally, the image recognition system is divided into 2 types, namely: a feature-based system and an image-based system. In the first system, features extracted from the attention image components, nose, mouth, etc. which are then modeled geometrically to determine the relationship between these features. Whereas in the second system using image pixels which are then represented in certain methods such as Principal Component Analysis, wavelet transformation, etc. which is used for training the system and image identification classification.

Feature extraction may be a process for obtaining characteristics that distinguish a face sample from another face sample. Reliable feature extraction techniques are the main key in solving pattern recognition problems because of the principal component analysis method (PCA) used for face recognition. The PCA method aims to project data in the direction that has the greatest variation (indicated by the eigenvector corresponding to the largest eigenvalues of the covariance matrix. The weakness of the tactic is a smaller amount optimal within the separation between classes. There is another method called Linear Discriminant Analysis (LDA) for face recognition. This method tries to seek out a linear subspace that maximizes the separation of two pattern classes consistent with Fisher Criterion JF. This can be obtained by minimizing the distance of the within-class Sw distribution matrix and maximizing the split matrix spacing between the $\mathrm{Sb}$ classes simultaneously resulting in a maximum Fisher Criterion JF. Fisher Linear Discriminant finds the subspaces where classes are linearly separated by maximizing the Fisher Criterion JF. If the data dimension is much higher than the number of training samples will cause $\mathrm{Sw}$ to be singular. This is a weakness of the LDA method.

Later, the fisherface method is introduced for face recognition. This method is a combination of PCA and LDA methods. The PCA method is used to solve singular problems by reducing the dimensions before being used to perform the LDA process. But the weakness of this method is that when the PCA dimension reduction process will cause some loss of discriminant information useful within the LDA process.

The data used in this study is the image of the CK's face. Face images of people of CK used as data in this study because also there has been no research data in the form of face images of the CK. Another reason is that the images of the face of the $\mathrm{CK}$ are generally very similar so there is a possibility of influence on the success of the method used in this study.

\section{Algorithm of FisherFaces:}

Let $\mathrm{X}$ be a random vector with samples drawn from $\mathrm{c}$ classes:

$$
\begin{array}{rr}
X= & \left\{\mathrm{X}_{1}, \mathrm{X}_{2}, \ldots, \mathrm{X}_{\mathrm{c}}\right\} \\
\mathrm{X}_{\mathrm{i}}= & \left\{\mathrm{x}_{1}, \mathrm{x}_{2}, \ldots, \mathrm{x}_{\mathrm{n}}\right\}
\end{array}
$$

The scatter matrices $S_{-}\{B\}$ and $S_{-}\{W\}$ are calculated as:

$$
\begin{array}{ll}
\mathrm{S}_{\mathrm{B}}= & \sum_{\mathrm{i}=1}^{\mathrm{c}} \mathrm{N}_{\mathrm{i}}\left(\mu_{\mathrm{i}}-\mu\right)\left(\mu_{\mathrm{i}}-\mu\right)^{\top} \\
\mathrm{S}_{W}= & \sum_{\mathrm{i}=1}^{\mathrm{c}} \sum_{\mathrm{x}_{\mathrm{j}} \in \mathrm{X}_{\mathrm{i}}}\left(\mathrm{x}_{\mathrm{j}}-\mu_{\mathrm{i}}\right)\left(\mathrm{x}_{\mathrm{j}}-\mu_{\mathrm{i}}\right)^{\top}
\end{array}
$$

, where $\mu$ is the total mean:

$$
\mu=\frac{1}{N} \sum_{i=1}^{N} x_{i}
$$

And $\mu_{i}$ is the mean of class $i \in\{1, \ldots, c\}$ :

$$
\mu_{i}=\frac{1}{\left|X_{i}\right|} \sum_{x_{j} \in X_{i}} x_{j}
$$

Fisher's classic algorithm now looks for a projection W, that maximizes the class separability criterion:

$$
W_{\mathrm{opt}}=\arg \max _{W} \frac{\left|W^{\top} S_{\mathrm{B}} W\right|}{\left|\mathbf{W}^{\top} S_{W} W\right|}
$$

A solution for this optimization problem is given by solving the General Eigenvalue Problem:

$$
\begin{array}{rrr}
\mathrm{S}_{\mathrm{B}} v_{\mathrm{i}} & = & \lambda_{\mathrm{i}} \mathrm{S}_{w} v_{\mathrm{i}} \\
\mathrm{S}_{\mathrm{W}}^{-1} \mathrm{~S}_{\mathrm{B}} v_{\mathrm{i}}= & \lambda_{\mathrm{i}} v_{\mathrm{i}}
\end{array}
$$

The optimization problem can then be rewritten as:

$$
\begin{array}{lr}
W_{\text {pca }}= & \arg \max _{W}\left|W^{\top} S_{\mathrm{T}} W\right| \\
W_{\text {fld }}= & \arg \max _{W} \frac{\left|W^{\top} W_{\text {pca }}^{\top} S_{\mathrm{B}} W_{\text {pca }} W\right|}{\left|W^{\top} W_{\text {pca }}^{\top} S_{W} W_{\text {pca }} W\right|}
\end{array}
$$

The transformation matrix $\mathrm{W}$, that projects a sample into the (c-1)-dimensional space is then given by:

$$
W=W_{\text {fld }}^{\top} W_{\text {pca }}^{\top}
$$

\section{FisherFaces Process:}

- Retrieve data: Collection of data is done in the form of face images. Collection can be done using photographs already saved or from a webcam. The face must be fully visible and must be facing forward. 
- Image Processing Process: This process is divided into two stages: preprocessing stage and processing stage which includes feature extraction and image recognition.

I. Image Preprocessing: Getting images using the camera or saved images and conversion from RGB to grayscale. Image data is divided into training and test data.

II. Image Processing: Fisherface method will be applied to generate a feature vector of facial image data used by the system and then to match vector of traits of training image with vector characteristic of the test image is done by using Euclidean distance formula.

- Feature generation process: Features of the faces are extracted.

Data images:

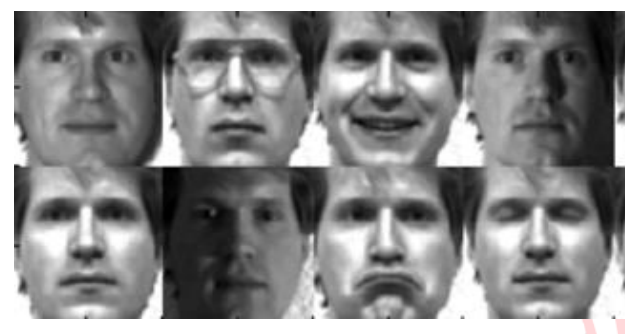

Fig: 7

Training images:
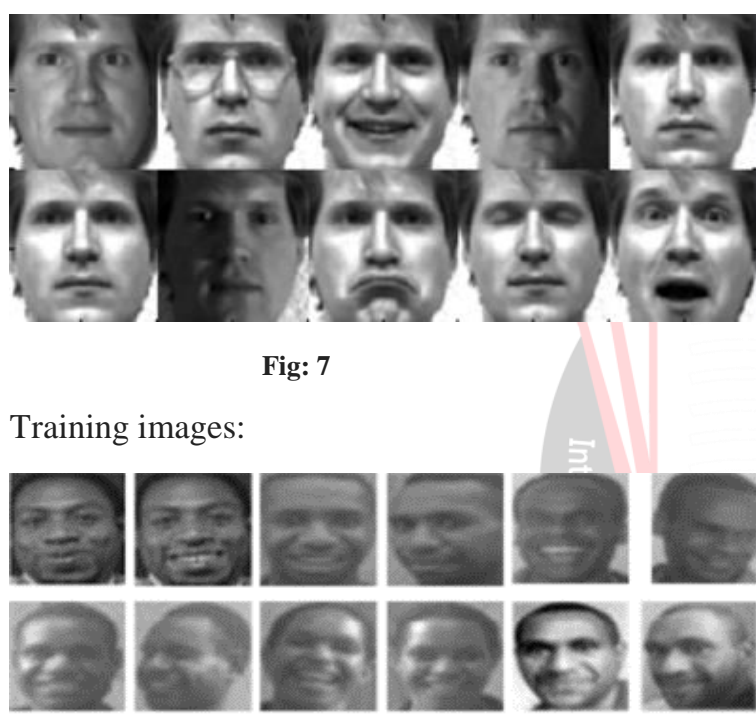

20
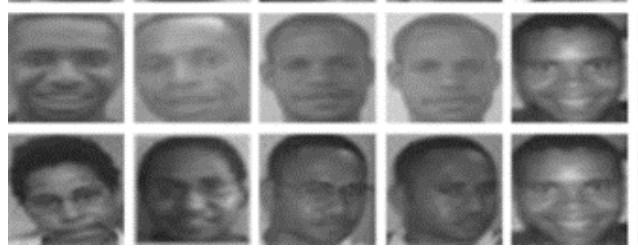

Fig: 8

Testing images:
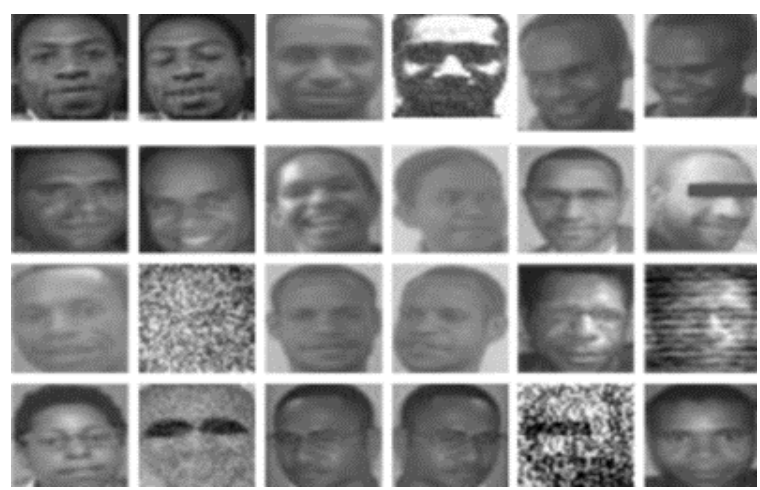

Fig:9
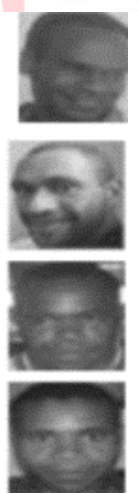

\section{Image Recognition Process:}

After the training process is successfully done, the next stage is to carry out the image recognition process.

The goal is how big the system successfully recognizes the test image or testing properly. The following are the results of the image recognition process performed by the system.

1)The training image and testing image both are the same. The purpose of image training is that the same because the test image that's the test image employed by the system within the recognition process is that the same because of the training image used at the time the training process, both in terms of expression, position and image condition. The number of facial images utilized in this process is 250 images. The test results show that the system is capable of successful identification of the test image correctly up to $100 \%$.

\section{2)The training image is not the same as the}

\section{Testing image.}

In this test, the testing image used is not the same as the training image used during the training, whether viewed in terms of expression, position, and condition of the image; but the test image and therefore the training image still come from the image of an equivalent person's face. The test results show that the system is capable of successful identification of the test image correctly up to $93 \%$.

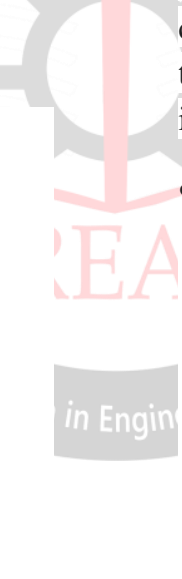

- Results:

\begin{tabular}{ccc}
\hline Training & Testing Image & Result \\
\hline & $\begin{array}{c}\text { recognized } \\
\text { correctly }\end{array}$ & $\begin{array}{c}\text { recognized } \\
\text { correctly }\end{array}$ \\
& & $\begin{array}{c}\text { recognized } \\
\text { correctly }\end{array}$ \\
& & $\begin{array}{c}\text { recognized } \\
\text { correctly }\end{array}$ \\
& &
\end{tabular}

Table:1 


\subsection{Room Rotation:}

The paper put forward the concept of room rotation to get the view of different directions from home. For room rotation purposes it has used motor, switch (button), and motor driver to control the motor. The room rotates when the person presses the button and it rotates until that person releases the switch. The room can rotate up to 360 degrees and gives the view of all the directions.

\subsection{Dynamic Facade:}

The dynamic facade is a concept used to control the sunlight intensity coming from window glass in the home/room. When sun rays hit the surface of the window glass, the facade opens to control light/ray intensity coming from the sun. Here the system has designed foam sheet facade and use MCP3208 IC, LDR, motor, and motor driver for the mechanism of the facade. LDR (Light Dependent Resistor) is also called photoresistor as it is used in light varying sensor circuits and also used in light and dark activated switching circuits. LDR generates the analog value as its output according to light value sensed. This generated analog output value is then given to MCP3208 IC and it converts this analog valuelsignal to a digital signal and sends it to the raspberry pi. According to the value, opening and closing action of the facade takes place.

\subsection{ThingsBoard:}

ThingsBoard is an open-source platform for data collection, processing, visualizing, and device management. It is a web application that collects all the data from home appliances and stores it. ThingsBoard permits users to access and control appliances from anywhere in the world and place track on the data collected. Data transmission between ThingsBoard and user is done through MQTT (Message Queuing Telemetry Transport) protocol so that anyone can access home appliances from anywhere in the world.

\section{CONCLUSION}

The system makes use of the internet to control home appliances. The proposed system can be one of the best solutions to the previous methods used to automate the house. This paper aims to ease the life of humans and also to save the time of people so that they can invest their time in other important work.

\section{FUTURE SCOPE}

The used system can be implemented on a large scale like huge buildings, offices, schools, etc. By doing more research on this, engineer can build a smart door that can open from eye detection. And also, voice recognition can be used to improve the modules implemented before.

\section{REFERENCES}

[1] Anmol Uppal, Shweta Tyagi, Rishi Kumar, Seema Sharma, "Emotion Recognition and Drowsiness Detection Using Python". 9thinternationalconference On Cloud computing, Data Science\& Engineering (Confluence) 2019IEEE.

[2] Sadi Mahmud, Safayet Ahmed, Kawshik Shikder" A Smart Home Automation and Metering System Using Internet of Things (IoT)." 2019 International Conference on Robotics, Electrical, and Signal Processing Techniques (ICREST).

[3] Ritvik Iyer, Antara Sharma, "IoT Based Home Automation System with Pattern Recognition.” International Journal of Recent Technology and Engineering (IJRTE) ISSN: 2277-3878, Volume-8 Issue-2, July 2019.

[4] Mustamin Anggo and La Arapu. "Face Recognition Using Fisherface Method." Journal of Physics: Conference Series, Volume 1028, 2nd International Conference on Statistics, Mathematics, Teaching, and Research 2017 9-10 October 2017, Makassar, Indonesia, 1 June 2018.

[5] Dr. Riham Nady, "Dynamic Facades Environmental Control Systems for Sustainable Design”. Volume 3 Issue 1, Special Issue, March 2017 ISSN 2356-8569.

[6] Kalyani Pampattiwar, Mit Lakhani, Rinisha Marar, And Rhea Menon." Home Automation Using Raspberry Pi Controlled Via an Android Application" International Journal of Current Engineering and Technology, Vol.7, No.3 (June 2017).

[7] B. Pandya, M. Mehta, N. Jain, "Android Based Home Automation System Using Bluetooth \& Voice Command", International Research Journal of Engineering and Technology (IRJET), Vol. 03 Issue. 03,Pp.609-611, 2016.

[8] Satish Palaniappan, Naveen Hariharan, Naren $\mathrm{T}$ Kesh, Vidhyalakshimi S, And Angel Deborah. S, "Home Automation Systems A Study", International Journal Of Computer Applications (0975 - 8887), Vol.116, Issue.11, April 2015.

[9] D. Pavithra, R. Balakrishnan, "IoT Based Monitoring and Control System for Home Automation", Global Conference on Communication Technologies (GCCT), Pp.169-173, 2015.

[10] Manish Kumar And Ramandeep Singh, "HOME Appliance Controlling Using ZIGBEE On ATMEGA128 Hardware Platform”, International Journal of Research in Engineering and Technology (IJRET), Vol. 03, Issue. 07, Pp. 469-472, Jul-2014.

[11] Ali M., V Laskamp J.H.A, Eddiny N.N., Falconer B., And Oram C., "Technical Development and Socioeconomic Implications of The Raspberry Pi as A Learning Tool in A Developing Countries" 5thcomputer Science and Electronic Engineering Conference (CEEC), Pp. 103-108,2013.

[12] Hsien-Tang Lin "Implementing Smart Homes with Open Source Solutions", International Journal of Smart Home, Vol. 07, Issue. 4, Pp.289- 295, July 2013.

[13] Rajeev Piyare And Seong Ro Lee, "Smart Home-Control and Monitoring System Using Smart Phone", ICCA, ASTL, Vol. 24, Pp.83862013.

[14] Faisal Baig, Saira Beg, Muhammad Fahad Khan, "Controlling Home Appliances Remotely Through Voice Command", International Journal of Computer Applications, Vol.48, Issue. 17, June 2012.

[15] Basil Hamed, "Design \& Implementation of Smart House Control Using LabVIEW", International Journal of Soft Computing and Engineering (IJSCE), Vol.01, Issue. 06, Pp. 98-103, January 2012.

[16] R. Piyare, And Tazil, M, "Bluetooth Based Home Automation System Using Cell Phone", IEEE 15thinternational Symposium on Consumer Electronics, 2011. 